\section{Alternative splicing of KLF6 associated with increased risk of prostate cancer}

One of the strongest risk factors for prostate cancer is family history of disease, but the predisposing genetic factors are unknown. KLF6 suppresses cell growth through p53independent transactivation of $\mathrm{p} 21$, and mutations leading to the inactivation or loss of KLF6 have been linked to other human cancers. Narla et al. investigated the possibility that inherited KLF6 mutations or polymorphisms exist that may increase prostate cancer risk.

Direct sequencing of genomic DNA isolated from blood revealed one very frequent polymorphism, IVS $\delta$ A; IVS1-27 G >A. To identify a possible association between this single nucleotide polymorphism and prostate cancer risk, germline DNA was genotyped from 3,411 geographically diverse men from three independent institutions, including 1,253 sporadic cancer patients, 882 familial cancer patients and 1,276 control men. It was found that there was a significant association between IVS $\delta A$ and cancer in both sporadic and familial cancer.

Using KLF6 minigene constructs in cultured cells, it was established that KLF6 was alternatively spliced, that there was an overabundance of KLF6 splice variants to wild type in tumor tissue, and that IVS $\delta A$ was consistently associated with enhanced KLF6 alternative splicing and variant protein expression. Complementary DNA sequence analysis confirmed the presence of three alternatively spliced KLF6 gene transcripts. These findings led to the suggestion that IVS $\delta A$ effected a change in wild-type KLF6 tumor suppressor gene expression and function. A splicing enhancer motif prediction program showed that the IVS $\delta A$ allele generates a functional SRp40 binding sequence, and studies with minigene constructs showed that spliced forms of KLF6 antagonize wildtype KLF6 effects on p21 and cell growth.

The authors note that the critical balance between the growth-suppressive and growthpromoting forms of KLF6 can profoundly influence prostate biology and cancer risk.

Original article Narla G et al. (2005) A germline DNA polymorphism enhances alternative splicing of the KLF6 tumor suppressor gene and is associated with increased prostate cancer risk. Cancer Res 65: 1213-1222

\section{A new role for sirolimus: regression of Kaposi's sarcoma in kidney-transplant recipients}

Immunosuppression is thought to be important in the natural history of Kaposi's sarcoma, and the disease is 500 times more prevalent in patients who have received a solid-organ transplant than in the general population. In such patients, the skin lesions associated with Kaposi's sarcoma can be controlled by reducing immunosuppressive therapy, but this increases the risk of graft rejection.

The immunosuppressive drug sirolimus (rapamycin) is reported to inhibit tumorigenesis by suppressing VEGF expression and disrupting the Akt-p70S6 signaling pathway; therefore, this compound has therapeutic potential for immunosuppression-related malignancies. Stallone and co-workers have recently investigated the antineoplastic properties of sirolimus in kidney-transplant recipients with Kaposi's sarcoma.

Between October 2001 and March 2004, 15 kidney-graft recipients (mean age 48.7 years) on identical immunosuppressive drug regimens were enrolled in the study. All patients were HIV negative. Immunosuppressive therapy was changed from cyclosporine, mycophenolate mofetil and prednisone to sirolimus and prednisone when Kaposi's sarcoma was diagnosed. Biopsies of lesions and normal skin were taken at diagnosis and analyzed for VEGF, Flk-1/KDR protein, phosphorylated Akt and p70S6 kinase, which were all found to be present in significantly higher levels in Kaposi's sarcoma cells than in normal skin cells. After 3 months of sirolimus therapy, cutaneous lesions were undetectable in all patients. Biopsies taken from the site of the original lesion, 6 months after sirolimus therapy was initiated, confirmed that all patients had attained clinical remission of Kaposi's sarcoma with no incidents of graft rejection. The authors conclude that sirolimus is effective in treating Kaposi's sarcoma in kidneytransplant patients, while maintaining an immunosuppressive effect.

Original article Stallone G et al. (2005) Sirolimus for Kaposi's sarcoma in renal-transplant recipients. N Engl J Med 352: $1317-1323$

\section{GLOSSARY}

VEGF

Vascular endothelial growth factor; a soluble factor that stimulates vascular permeability and blood vessel growth 